\title{
Overview of SHI Induced Track Morphology in Crystalline Non-Metals from Direct Observation with TEM
}

\author{
J.H. O'Connell ${ }^{a} *$, V.A. Skuratov ${ }^{b}$, A. Janse van VuUren ${ }^{a}$ And R.A. Rymzhanov ${ }^{b}$ \\ ${ }^{a}$ Centre for HRTEM, Nelson Mandela University, P.O. Box 77000, Port Elizabeth 6031, South Africa \\ ${ }^{b}$ Flerov Laboratory for Nuclear Research, Joint Institute for Nuclear Research, \\ Joliot-Curie 6, 141980 Dubna, Moscow region, Russia
}

\begin{abstract}
We studied the track morphology of $167 \mathrm{MeV}$ Xe ion irradiated amorphizable and non-amorphizable single crystals, in the non-overlapping track regime, by direct observation with transmission electron microscopy. Simulation of the early stages of the interaction shows transient melting for all materials while the interaction of closely spaced tracks that do not overlap was found to increase with a material's resistance to track formation. This lead to track density saturation and delayed amorphization of the bulk crystal. We propose a structure dependent recrystallisation rate as the main factor determining the size and morphology of ion tracks.
\end{abstract}

DOI: 10.12693/APhysPolA.136.233

PACS/topics: $60.80 .-\mathrm{x}, 61.80 . \mathrm{Az}$

\section{Introduction}

Swift heavy ion (SHI) induced crystal damage is usually associated with so-called ion tracks. These consist of lines of disordered material which was exposed to high levels of energy deposition during passage of a SHI. The material response during such interactions is typically described by a temperature spike model [1] which is dependent on the thermomechanical properties of the target material as well as the electronic energy loss $\left(S_{e}\right)$ of the ion in question. This model depends strongly on electron-phonon coupling coefficients which are not trivial to obtain. Measurements of experimental track diameters can be fed back into models in order to estimate these coefficients, but this in turn depends on a robust, reliable diameter measurement which itself is not well defined for certain materials where tracks do not manifest as continuous amorphous cylinders.

Moreover, at higher fluences where track overlap occurs, complete amorphization may occur $[2,3]$ as well as synergistic effects such as nanocrystal formation $[4,5]$ and track annealing by subsequent ions $[6,7]$. Struder et al. [8] reviewed the available transmission electron microscopy (TEM) data regarding ion tracks in various materials. They noted the absence of track formation in pure crystalline semiconductors such as $\mathrm{Si}$ and Ge while $\mathrm{GeS}$ and $\mathrm{SiO}_{2}$ are amorphizable by SHI irradiation. High energy fullerene irradiation however does produce amorphous tracks in $\mathrm{Si}[9]$ but this represents the extreme case of high $S_{e}$ and simultaneous low ion velocity. Naguib and Kelly [10] considered data on 72 substances irradiated to fluences ranging from $10^{13}$ to over $10^{17}$ ions $/ \mathrm{cm}^{2}$

*corresponding author; e-mail: joconnell@mandela.ac.za using low energy heavy ions. They proposed two criteria to determine amorphization probability by ion irradiation where amorphization was probable if either the ratio of (crystallization temperature)/(melting point) exceeds 0.3 or when the material ionicity is less or equal to 0.47 . Although these criteria gave satisfactory results for the tested materials, the interaction of SHI's with materials are fundamentally different from that of low energy heavy ions. In this paper, we describe the general morphology of SHI induced tracks in non-metallic crystals of varying structure and composition as observed by TEM in order to gain some insight on what governs the nature of SHI induced tracks.

\section{Experimental}

Irradiations were performed at room temperature using $167 \mathrm{MeV}$ Xe ions at the JINR cyclotron complex, Dubna, Russia. Target materials were single crystals of yttrium aluminium garnet $\left(\mathrm{Y}_{3} \mathrm{Al}_{5} \mathrm{O}_{12}-\mathrm{YAG}\right)$, yttrium iron garnet $\left(\mathrm{Y}_{3} \mathrm{Fe}_{5} \mathrm{O}_{12}-\mathrm{YIG}\right)$, yttrium aluminium perovskite $\left(\mathrm{YAlO}_{3}-\mathrm{YAP}\right), \alpha-\mathrm{Al}_{2} \mathrm{O}_{3}, \mathrm{TiO}_{2}$ (rutile), $\mathrm{CeO}_{2}$, yttrium stabilized zirconia (YSZ), and $\mathrm{MgO}$ and fluences were maintained in the non-track overlapping regime between $2 \times 10^{10}$ and $1 \times 10^{11}$ ions $/ \mathrm{cm}^{2}$. Ion flux homogeneity of better than $5 \%$ was achieved by horizontal and vertical beam scanning. After irradiation, target materials were thinned to electron transparency using an FEI Helios Nanolab 650 FIB in both cross-section and plan view geometries. Final thinning was performed at $5 \mathrm{kV}$ accelerating voltage and further polishing was performed down to $1 \mathrm{kV}$ to minimize Ga induces crystal damage. In all cases the ion range into the crystal was in excess of $10 \mu \mathrm{m}$ while plan view specimens were extracted within $1 \mu \mathrm{m}$ from the surface. The prepared lamellae were then imaged using a double $\mathrm{C}_{s}$ corrected JEOL ARM $200 \mathrm{~F}$ operating at $200 \mathrm{kV}$. 


\section{Results}

Figure 1 shows typical plan view annular bright field $(\mathrm{ABF})$ scanning transmission electron microscope (STEM) micrographs for amorphizable materials YAG, YIG, and YAP. YAG and YIG have essentially the same structure with Al substituted for Fe in YIG. The electronic stopping power of the Xe ions were slightly higher in YIG $(23.9 \mathrm{keV} / \mathrm{nm})$ than in YAG $(22.5 \mathrm{keV} / \mathrm{nm})$ and the result of these two affects is an increase in track diameter from $6.52 \pm 0.58 \mathrm{~nm}$ in YAG to $10.05 \pm 0.55 \mathrm{~nm}$ in YIG based on about 60 track measurements for each material. YAP has a considerably less complex structure with a much smaller unit cell and also a smaller track diameter of $3.75 \pm 0.66 \mathrm{~nm}$ despite having electronic stopping power between YIG and YAG at about $23 \mathrm{keV} / \mathrm{nm}$. From Fig. 1 it is clear that the track periphery in YAP is not as smooth as in YAG and YIG.
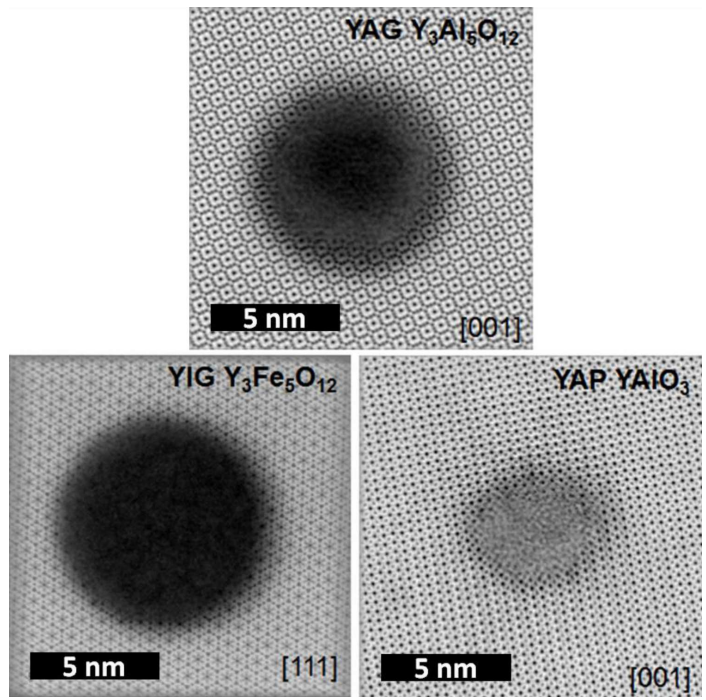

Fig. 1. ABF STEM micrographs of $167 \mathrm{MeV}$ Xe ion tracks in YAG, YIG and YAP.

Figure 2 shows cross-sectional ABF STEM micrographs of ion tracks in YAG to demonstrate the continuous cylindrical nature of these features. The irradiated surface in Fig. 2a is represented by the dark horizontal line along the centre of the micrograph. A faint hillock can be distinguished on top of the surface above the track. The bright contrast above the surface is due to electron beam deposited carbon (bright band just above the surface) and Ga beam deposited carbon (darker region at top of image) which was laid down during specimen preparation in the FIB. The lower magnification image in part (b) shows several continuous ion tracks. Some of the tracks exit the foil before touching the irradiated surface since the foil thickness was much larger that the track diameter and the thinnest part of the wedge shaped foil was at the top of the image. Similar cross-sectional track structure was found for YIG and YAP.
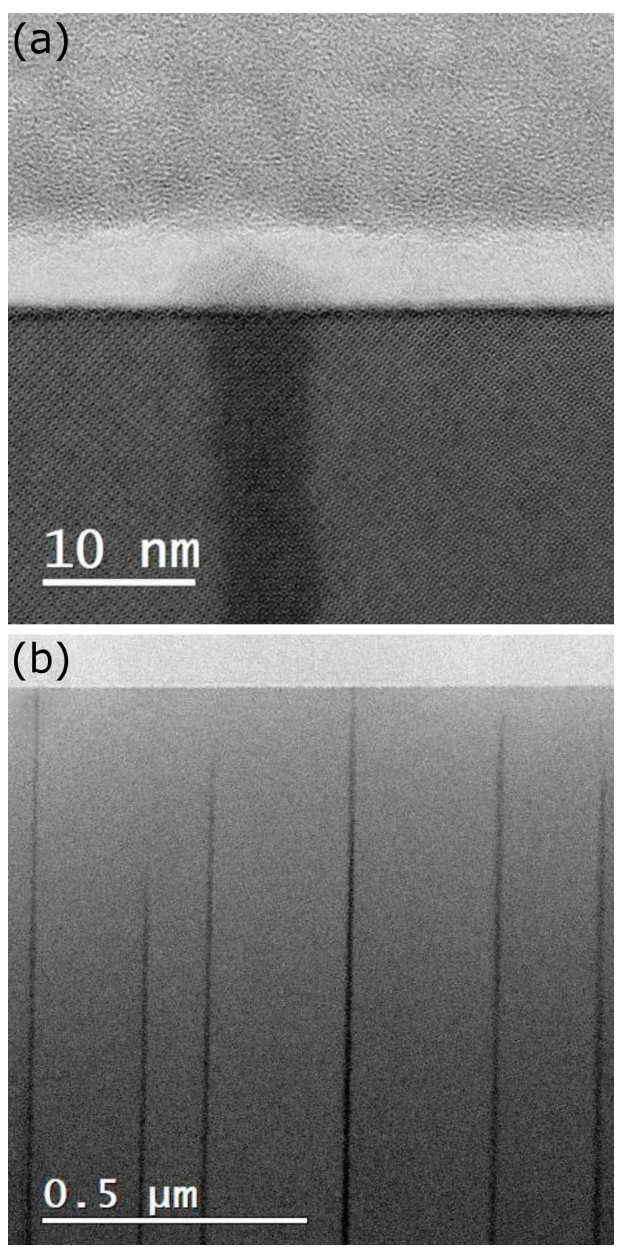

Fig. 2. Cross-sectional ABF STEM micrograph of an ion track in YAG showing the continuous nature of the track (a) and a lower magnification micrograph (b) showing several tracks.

Figure 3 shows plan view ABF STEM micrographs of closely spaced tracks in YIG and YAP. Little to no interaction between nearby tracks is observed in YIG while closely spaced tracks in YAP are connected by extended regions of defected crystal as can be seen from the dark strain contrast connecting the amorphous track cores. Closely spaced tracks in YAG behaved similarly to that of YIG with no noticeable interaction between individual tracks. The interaction of closely spaced tracks suggests an initial area of effect during ion impact significantly larger than the resulting track. This coupled with the slightly smaller than expected track diameter in YAP suggests that some recrystallization of the initial molten zone occurred.

Figure 4 shows HRTEM micrographs of individual $167 \mathrm{kV}$ Xe ion tracks in alumina $\left(S_{e} \approx 25 \mathrm{keV} / \mathrm{nm}\right)$ and rutile $\left(S_{e} \approx 24 \mathrm{keV} / \mathrm{nm}\right)$. Unlike the situation with the more complex YAG, YIG, and YAP, these tracks lack clear amorphous cores and manifest as discontinuous regions of strain along the ion path $[3,6]$. Defining 

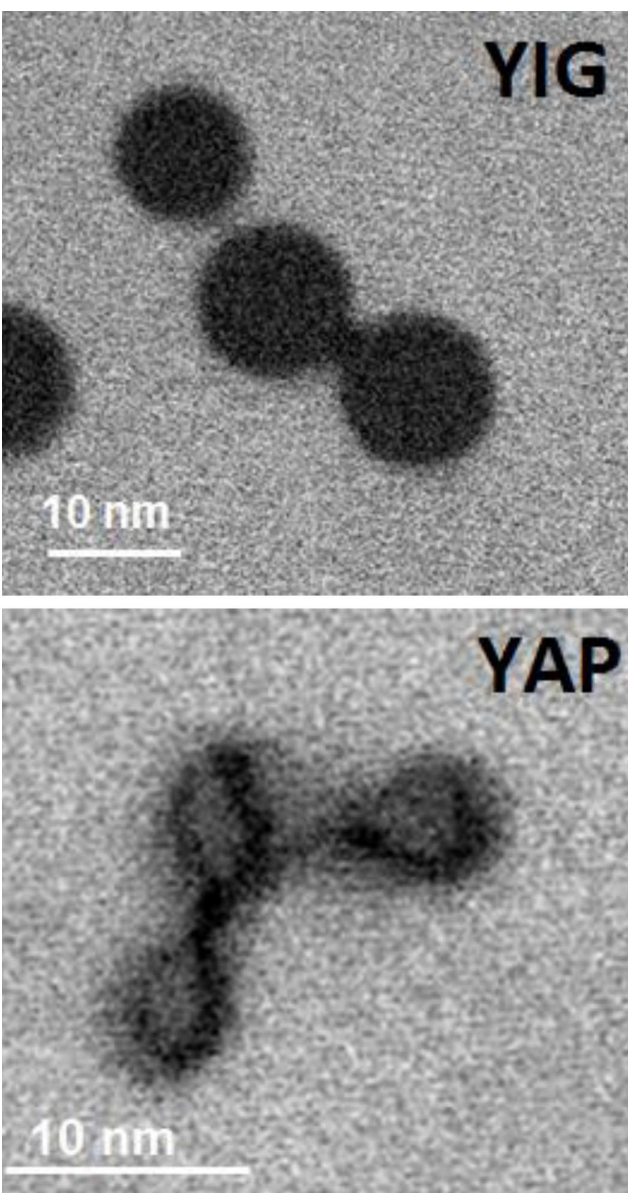

Fig. 3. ABF STEM micrographs of closely spaced tracks in YIG and YAP.

a meaningful "track diameter" in these materials through direct observation is complicated by the discontinuous nature of the track and the small size of individual features along the track $(\approx 2-4 \mathrm{~nm})$ since any plan view image is always a projection of several such features overlapping with unaffected crystal. In cross-section, the strain fields around individual track features can be seen from diffraction contrast as shown for alumina in the topmost micrograph in Fig. 5, but the large variability in size of these contrasting regions make quantification difficult. The left image in Fig. 5 shows a simulated ion track for $167 \mathrm{MeV}$ Xe in bulk alumina while the rightmost image is a crop of one of the tracks in the top image shown at the same magnification as the simulation. The simulation was performed using a hybrid approach that consists of a Monte-Carlo simulation (MC code TREKIS [11, 12]) of the electron kinetics, and molecular dynamics model of atomic dynamics using LAMMPS [13]. Very good agreement in the size and distribution of defected zones in the crystal can be seen.

Indirect methods such as the Rutherford backscattering in channelling configuration (RBS-C) can infer "effective" diameters by assuming the total measured disorder in the probed layer is the sum of identical cylindrical tracks but this assumption is clearly not valid
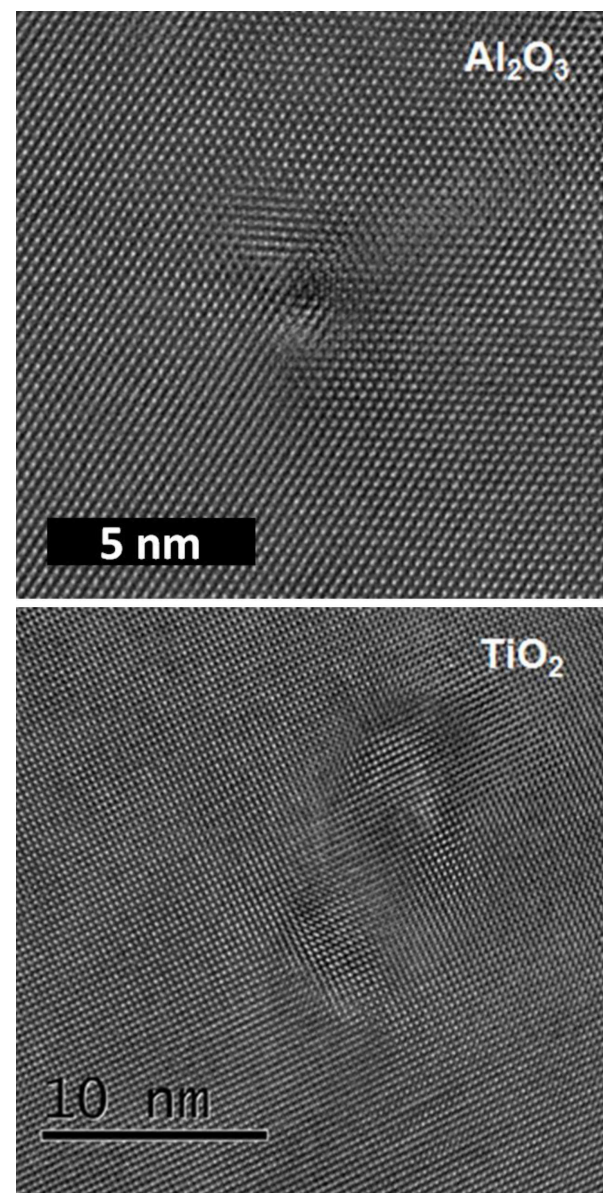

Fig. 4. Plan view HRTEM micrographs of $167 \mathrm{MeV}$ Xe in $\mathrm{Al}_{2} \mathrm{O}_{3}$ and $\mathrm{TiO}_{2}$.
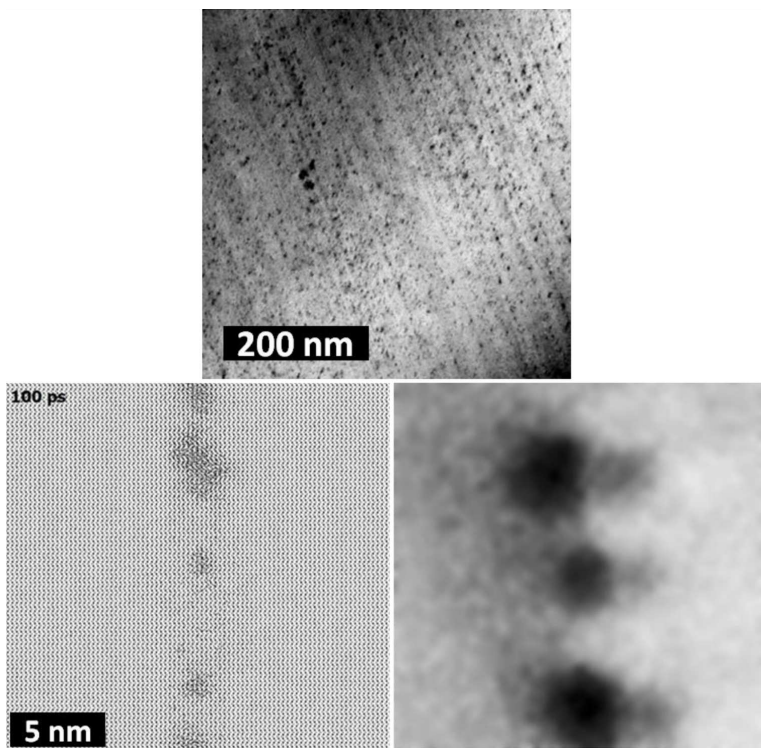

Fig. 5. BF TEM micrograph of Xe tracks in alumina (top) with white arrow indicating the ion direction. A simulated Xe track showing regions of distorted crystal (left) and a crop of one of the tacks from the left image at the same magnification and orientation as the simulation (right). 
for these materials. Also, it has been shown previously that the near surface regions of these materials exhibit significantly different track morphology than in the bulk [14].

The tracks in Fig. 4 appear only as visible distortions in the periodicity of the lattice image, similar results were obtained for $\mathrm{CeO}_{2}$ and YSZ, all of which have somewhat simpler unit cells than YAG, YIG, or YAP with only two atomic species and small unit cells. In these materials, closely spaced tracks have a strong influence on one another with younger tracks affectively annealing out older tracks almost completely. This leads to track density saturation $[6,7]$ and much delayed amorphization. Once again this suggests significant recrystallization of the initial molten zone during cooling, resulting in only discontinuous zones of distorted crystal.

No indication of track formation could be observed in $\mathrm{MgO}$. In all cases, the materials were stable in the electron beam during imaging. Nonetheless, care was taken to minimise the electron dose in the imaged areas prior to acquiring the micrographs. Focussing the electron beam on tracks in YAP caused them to partially recrystallize but only after several seconds of exposure to the high intensity beam. No track changes were observed for the other materials.

\section{Discussion}

Large, complex, multi-component crystals such as YAG, YIG, and to a lesser extent, YAP, produce continuous, amorphous tracks of cylindrical shape after SHI impact. Less complex structures such as alumina, rutile, YSZ, and ceria produce only discontinuous zones of distorted crystal of a few $\mathrm{nm}$ in size. $\mathrm{MgO}$ shows no visible signs of track formation at all. In all these materials, electronic stopping power of the incident ions was comparable and simulation predicts transient melting in all cases. In this case we refer to transient melting since the timescale involved is extremely short and thermal equilibrium is not reached. The loss of an ordered periodic structure at high temperature due to excitation by an energetic ion can be considered a "molten" state in this regard. This, together with the observation of track induced annealing of nearby tracks which increases as the material's resistance to track formation increases, suggests that recrystallization of the molten zone is the dominant factor controlling the final size and morphology of SHI induced tracks. A simple qualitative model considering unit cell complexity in size, structure, and number of atomic species as a measure of recrystallization ability seems reasonable. Crystals that are able to recrystallize within the cooling period of the transient thermal spike will have little remaining distortion which constitutes a track. Further work is planned to elaborate this model in a more quantifiable manner.

\section{Conclusion}

It was found that multi-component, complex structures tend to form mostly continuous, cylindrical, amorphous tracks of relatively large diameter. On the other hand, simpler structures tend to form discontinuous tracks composed of strained crystalline regions with the limiting case of $\mathrm{MgO}$ showing no discernible tracks to TEM. Interaction between nearby tracks that do not overlap suggests relatively large transiently molten zones during ion impact and we suggest the material's ability to quickly recrystallize during the cooling phase of the temperature spike as the controlling factor to track formation.

\section{References}

[1] M. Toulemonde, C. Dufour, A. Meftah, E. Paumier, Nucl. Instrum. Methods Phys. Res. B 166, 903 (2000).

[2] G. Szenes, Journal of Nuclear Materials 336, 81 (2005).

[3] J.H. O'Connell, V.A. Skuratov, A. Akilbekov, A. Zhumazhanova, A. Janse van Vuuren, Nucl. Inst. Meth. B 379, 200 (2016)

[4] J.M. Costantini, F. Ravel, F. Brisard, M. Caput, C. Cluzeau, Nucl. Inst. Meth. B 80, 1249 (1093).

[5] J.M. Costantini, S. Miro, F. Beuneu, M. Toulemonde, J. Phys. Condens. Matter 27, 496001 (2015).

[6] J.H. O'Connell, R.A. Rymzhanov, V.A. Skuratov, A.E. Volkov, N.S. Kirilkin, Nucl. Inst. Meth. B 374, 97 (2016).

[7] K. Yasuda, M. Etoh, K. Sawada, T. Yamamoto, K. Yasunaga, S. Matsumura, N. Ishikawa, Nucl. Instr. Meth. Phys. Res. B 314, 185 (2013).

[8] F. Studer , M. Hervieu, J.M. Costantini, M. Toulemonde, Nucl. Inst. Meth. B 122, 449 (1997).

[9] B. Canut, N. Bonardi, S.M.M. Ramos, S. Della-Negra, Nucl. Inst. Meth. B 146, 296 (1998).

[10] H. Naguib, R. Kelly, Rad. Eff. 25, 1 (1975).

[11] N.A. Medvedev, R.A. Rymzhanov, A.E. Volkov, J. Phys. D. Appl. Phys. 48, 355303 (2015).

[12] R.A. Rymzhanov, N.A. Medvedev, A.E. Volkov, Nucl. Inst. Meth. B 388, 41 (2016).

[13] S. Plimpton, J. Comput. Phys. 117, 1 (1995).

[14] J.H. O'Connell, V.A. Skuratov, Phys. Status Solidi B 11, 2144 (2016). 